SIR: Dr Al-Sheikhli correctly points out that I omitted from my Maudsley lecture three useful treatments for depression. In my preamble I indicated my intentions particularly to review choice among treatments and also to illustrate the two-way relationship between research and clinical practice. The field is large and I had perforce to limit myself to five common treatments. Each was chosen because there existed a large number of published treatment studies and because it exemplified some aspect of the interrelationship between practice and research. Lithium carbonate is well established as a prophylactic treatment for bipolar manic-depressive disorder and some unipolar patients certainly could have met my criteria for inclusion. Lithium augmentation is undoubtedly valuable but there have been fewer controlled studies. Psychosurgery also has a place in severe resistant depression although here, more conclusive research regarding efficacy and indications is still needed. This might be something which the College itself could take up: I understand that the Mental Health Act Commission has comprehensive records of patients who have received psychosurgery since the new Act.

Department of Psychiatry

University of Cambridge

Addenbrooke's Hospital

Cambridge CB2 $2 Q Q$

E. S. PAYKeL

\section{Anticholinergics: the consensus statement}

SIR: We are grateful to Barnes for his comment (Journal, March 1990, 156, 413) on our consensus statement (Journal, March 1990, 156, 412), because it gives us the opportunity to provide some information on the purposes and the underlying rationale of the series of such statements that our group is currently producing.

These papers approach some highly controversial topics in clinical psychopharmacology, providing for each a concise outline of the different views expressed in the literature and a list of recommendations agreed upon by all the members of the group.

The statements are directed not so much at researchers or experts in the field as at the large audience of clinicians working in psychiatric wards, out-patient units or community services. This is why they are very brief, without any data or even references: in fact, it is our experience that very busy practitioners prefer this format.

The aim is not, therefore, to provide an exhaustive review of the literature or of the opinions of different authors and their empirical support. Furthermore, it should be stressed that the statements are designed for worldwide dissemination (each of them will be translated into several languages), and the recommendations are aimed at psychiatrists in developing as well as industrialised countries.

Prophylactic use of anticholinergics in patients on long-term neuroleptic treatment was found to be a suitable topic for a consensus statement because: (a) the views expressed in the literature about this issue are very discordant; and (b) it is important for the World Health Organization (WHO) to receive a clear input about the usefulness of a class of medications which are largely prescribed to psychotic patients in industrialised countries, but have never been included by the WHO in the list of essential psychiatric drugs.

In order to produce the statement, several discussions were necessary since opinions were initially divergent within our group. We are not surprised, therefore, that Dr Barnes' views are not in perfect agreement with our final consensus. In this sense, we see Dr Barnes' comment as an addition rather than as a criticism of our statement and welcome it without any reservation.

The comment expands some of the points made in our paper, adding details and references. Dr Barnes argues that, on the basis of the available literature, "the issue seems to be far from resolved". He is perfectly right: actually, in the presence of an agreement in the literature, the topic would have not been selected.

We agree with Dr Barnes' points that some of the hazards and side-effects of anticholinergic drugs listed in the document, such as the contribution to hyperthermic episodes and the antagonism of the therapeutic effects of antipsychotics, "are relatively uncertain", and that short-term prophylactic use of anticholinergics in the early phase of neuroleptic treatment may sometimes be useful. His formulation amplifies the cautions already present in the statement.

According to Dr Barnes, "the statement suggests that short-term prophylactic treatment is particularly useful to avoid the development of akathisia". Clearly, we were not sufficiently explicit: our document simply mentions, when listing the arguments in favour of prophylatic treatment with anticholinergics, that these drugs have been claimed to be useful in avoiding the appearance of neurological manifestations (such as akinesia and akathisia) which may mimic psychopathological symptoms and therefore lead to an inappropriate increase of the neuroleptic dosage (see Kane, 1988). It is not surprising, however, that Dr Barnes, who rightly points out that anticholinergics have "an uncertain reputation" in akathisia, concludes that "one explanation for the 